\title{
Tratamento Radioterápico no Sistema Único de Saúde: uma Análise do Período 2012 a 2016
}

https://doi.org/10.32635/2176-9745.RBC.2018v64n4.194

Radiotherapy Treatment in the National Health System: an Analysis of the Period from 2012 to 2016

Tratamiento Radioterápico en el Sistema Único de Salud: un Análisis del Período 2012 a 2016

Jeane Glaucia Tomazelli'; Adriana Tavares de Moraes Atty²; Antônio Carlos Antunes Bertholasce ${ }^{3}$; Maria Beatriz Kneipp Dias ${ }^{4}$

Resumo

Introduçáo: A radioterapia é uma das modalidades terapêuticas no tratamento do câncer. Objetivo: Descrever o perfil epidemiológico e assistencial do tratamento radioterápico no Sistema Único de Saúde no período 2012-2016. Método: Estudo descritivo sobre as Autorizaçôes de Procedimentos de Alta Complexidade em Oncologia do Sistema de Informaçáo Ambulatorial. Avaliaram-se o quantitativo de Cartão Nacional de Saúde nas autorizaçóes de radioterapia do tipo inicial, o número de autorizaçôes por cartão, as neoplasias mais frequentes, o número de campos de radioterapia aplicados, a idade mediana e a finalidade do tratamento por estabelecimento habilitado em oncologia. Resultados: Identificaram-se 509.708 cartões com uma razão de duas autorizaçốes/cartão. Mama (24,3\%) e próstata (18,5\%) foram as neoplasias mais frequentes. O número de campos por neoplasia foi superior ao preconizado. A mediana de idade foi menor para encéfalo (48 anos) e maior para próstata (70 anos). Maior parte do tratamento $(85,2 \%)$ foi para finalidade náo paliativa e foram realizadas em Centro de Assistência de Alta Complexidade em Oncologia com serviço de Oncologia Pediátrica. Conclusáo: $\mathrm{O}$ estudo demonstrou um incremento no número de casos/ano tratados com radioterapia, evidenciando a importância dessa modalidade terapêutica. Estudos que caracterizem o perfil dos casos em tratamento de câncer são necessários para qualificar a gestáo da rede de atenção a saúde.

Palavras-chave: Radioterapia; Neoplasias; Sistema Único de Saúde; Sistemas de Informação.

\section{Abstract}

Introduction: Radiotherapy is one of the therapeutic modalities in the treatment of cancer. Objective: To describe the epidemiology and assistance profile the radiotherapy treatment in the Unified Health System in the period 2012-2016. Method: Descriptive study about Authorization of Procedures of High Complexity in Oncology of the Outpatient Information System. The quantitative of national health card, the number of authorization by card, the most frequent neoplasia cases, the number of fields for the treatment of neoplasia, the average age of neoplasia treatment and the aim of treatment by licensed facilities in oncology. Results: 509,708 CNS were identified with a ratio of two authorization/card. Breast cancer $(24.3 \%)$ and prostate cancer $(18.5 \%)$ were the most frequent neoplasms. The number of fields per neoplasia was higher than the one recommended. Average age was lower for encephalon ( 48 years) and longer for prostate (70 years). Most of the treatment $(85.2 \%)$ was for non-palliative purposes and were performed in Center of High Complexity in Oncology with Pediatric Oncology Service. Conclusion: The study demonstrated an increase in the number of cases/ year treated with radiotherapy, evidencing an important of this therapeutic modality. Studies that characterize the profile of cases in cancer treatment are necessary to qualify the management of the health care network.

Key words: Radiotherapy; Neoplasms; Unified Health System; Information Systems.

\section{Resumen}

Introducción: La radioterapia es una de las modalidades terapéuticas en el tratamiento del cáncer. Objetivo: Describir el perfil epidemiológico y asistencial del tratamiento radioterápico en el Sistema Único de Salud en el período 2012-2016. Método: Estudio descriptivo sobre las Autorizaciones de Procedimientos de Alta Complejidad en Oncología del Sistema de Información Ambulatoria. Se evaluó el cuantitativo de tarjeta nacional de salud en las autorizaciones de radioterapia del tipo inicial, el número de autorizaciones por tarjeta, las neoplasias más frecuentes, el número de campos de radioterapia aplicados, la edad mediana y la finalidad del tratamiento por establecimiento habilitado en el establecimiento oncología. Resultados: Se identificaron 509.708 tarjetas con una razón de dos autorizaciones/tarjeta. Mama (24,3\%) y próstata $(18,5 \%)$ fueron las neoplasias más frecuentes. El número de campos por neoplasia fue superior al preconizado. La mediana de edad fue menor para el encéfalo (48 ańos) y mayor para la próstata (70 ańos). La mayor parte del tratamiento $(85,2 \%)$ fue para fines no paliativos y fueron realizadas en Centro de Alta Complejidad em Oncología con servicio de Oncología Pediátrica. Conclusión: El estudio demostró un incremento del número de casos/año tratados con radioterapia, evidenciando un importante de esta modalidad terapéutica. Los estudios que caracterizan el perfil de casos en tratamiento de cáncer son necesarios para calificar la gestión de la red de atención a la salud. Palabras clave: Radioterapia; Neoplasias; Sistema Único de Salud; Sistemas de Información.

\footnotetext{
1 Instituto Nacional de Câncer José Alencar Gomes da Silva (INCA). Rio de Janeiro (RJ), Brasil. Orcid iD: https://orcid.org/0000-0002-2472-3444

${ }^{2}$ INCA. Rio de Janeiro (RJ), Brasil. Orcid iD: https://orcid.org/0000-0003-2271-746X

${ }^{3}$ INCA. Rio de Janeiro (RJ), Brasil. Orcid iD: https://orcid.org/0000-0002-8426-3128

${ }^{4}$ INCA. Rio de Janeiro (RJ), Brasil. Orcid iD: https://orcid.org/0000-0002-5847-9830

Endereço para correspondência: Jeane Glaucia Tomazelli. Rua Marquês de Pombal, 125, 70 andar - Centro. Rio de Janeiro (RJ). CEP $22230-240$.

E-mail: jtomazelli@inca.gov.br
} 


\section{INTRODUÇÃO}

A radioterapia $(\mathrm{RxT})$ é uma importante modalidade terapêutica no tratamento do câncer, seja isoladamente ou em conjunto com a cirurgia ou quimioterapia. É a modalidade de maior investimento no tratamento oncológico, sendo a ampliaçáo de sua oferta uma estratégia nacional importante para o controle do câncer, estruturando a capacidade de tratamento e fornecendo cuidado adequado aos pacientes ${ }^{1}$.

De acordo com a International Atomic Energy Agency (Iaea), fórum intergovernamental central do mundo para cooperação científica e técnica na área nuclear, cerca de $50 \%$ a $60 \%$ dos casos oncológicos, em países de baixa e média rendas, necessitarão de radioterapia ${ }^{2,3}$. Além disso, estima-se que $25 \%$ dos casos necessitarão de novo tratamento por $\mathrm{RxT}^{4,5}$.

O tratamento com RxT ocorre por meio de irradiação local ou locorregional de áreas do corpo do paciente com neoplasia. Pode ser realizada por uma fonte de irradiação longe do organismo, pelos equipamentos: acelerador linear, unidade de cobaltoterapia e de ortovoltagem, sendo, então, denominada externa. Caso seja feita por uma fonte em contato com o corpo, como nos procedimentos de braquiterapia e betaterapia, é denominada RxT interna ou de contato ${ }^{6,7}$.

A radioterapia externa pode ser de megavoltagem (acelerador linear, unidade de cobaltoterapia) e ortovoltagem (roentgenterapia), mas os aceleradores lineares geram fótons de energia muito maiores; logo, liberam dose menor para a pele do paciente do que a unidade de cobaltoterapia ${ }^{7}$. Já a braquiterapia pode ser de baixa e alta doses ${ }^{6}$.

O Sistema Único de Saúde (SUS) prevê o tratamento integral aos casos diagnosticados com câncer em estabelecimentos habilitados pelo Ministério da Saúde (MS), como Unidade de Assistência de Alta Complexidade em Oncologia (Unacon) ou Centro de Assistência de Alta Complexidade em Oncologia (Cacon) ${ }^{8}$. Independente do tipo de habilitação, os estabelecimentos devem garantir a cirurgia e a quimioterapia, mas o tratamento radioterápico é facultativo para as Unacon. O que não exime as unidades sem radioterapia de referenciar formalmente os pacientes que necessitem dessa modalidade terapêutica para os estabelecimentos que o possuam.

O tratamento radioterápico no SUS, classificado como tratamento ambulatorial de alta complexidade, depende de autorização prévia: Autorização de Procedimentos de Alta Complexidade (Apac). A Apac está sujeita a várias regras, descritas no Manual de Bases Técnicas para Oncologia ${ }^{6}$, que visam a garantir a qualidade do tratamento e a segurança do paciente.
Para a Apac de radioterapia, é necessário registrar o Planejamento Terapêutico Global (PG) com definição da data de início e fim do tratamento, da finalidade da radioterapia, a descrição da área irradiada, da topografia segundo a Classificação Estatística Internacional de Doenças e Problemas Relacionados com a Saúde (CID) e o número total de campos ${ }^{6}$.

A mensuração da radioterapia externa no SUS é por campos, enquanto a braquiterapia de alta dose é por inserção. O campo é o número de incidências diárias em que se aplica a radioterapia externa, variando de um a seis em uma área externamente delimitada ${ }^{6}$. O tempo médio de tratamento é de quatro a cinco semanas, sendo possível utilizar intervalos de tempo com doses totais menores ou maiores ${ }^{6,9}$.

O número máximo de campos de radioterapia expressa a dose total prevista para a radiaçáo e varia segundo a localizaçáo primária do tumor. O Manual de Bases Técnicas da Oncologia $a^{6}$ apresenta um limite máximo de campos para cada neoplasia, sendo a neoplasia de próstata a que admite o maior número de campos (144 campos). E ainda determina que somente a RxT de finalidade curativa e, quando exclusiva, a de finalidade paliativa podem alcançar os números máximos de campos; e que é permitido número de campos superior ao estabelecido quando a finalidade terapêutica for anti-hemorrágica ou antiálgica e ainda em tratamento de resgate de tumor residual localizado (tratamento de exceção).

Em razão do exposto, o objetivo deste estudo é descrever o perfil epidemiológico e assistencial do tratamento radioterápico no SUS, a partir das informações das Apac, disponíveis na base do Sistema de Informação Ambulatorial do SUS (SIA-SUS), e à luz do Manual de Bases Técnicas da Oncologia ${ }^{6}$, visando a conhecer as características dos casos de câncer que demandaram essa terapêutica, bem como dos estabelecimentos que realizaram o tratamento.

\section{MÉTODO}

Foi realizado um estudo descritivo exploratório sobre as Apac em Oncologia para realização de radioterapia, registradas no Brasil no período de 2012 a 2016.

Foram utilizadas informaçóes das Apac em Oncologia para radioterapia (Apac-RxT) do SIA-SUS. Construiu-se um banco de dados a partir dos arquivos mensais, do período definido, por Unidade de Federaçáo. O banco foi restrito às Apac-RxT do tipo inicial ${ }^{10}$, por ano de realização do tratamento. Após análise preliminar da distribuição de frequência das neoplasias, as Apac-RxT iniciais foram agrupadas por tipo de neoplasia mais frequentes, sendo as neoplasias de menor frequência agrupadas como 'outras neoplasias'. 
As Apac únicas - indicadas para procedimentos que não admitem tratamento contínuo, e as Apac do tipo continuidade - que ocorrem quando a autorização é apresentada no segundo mês de realizaçáo do tratamento e mantêm o mesmo código de autorizaçáo, Cartáo Nacional de Saúde (CNS), procedimento e CID da Apac inicial, foram excluídas do estudo.

Também foram excluídas as Apac-RxT iniciais duplicadas, nas quais os registros eram idênticos em todas as variáveis do banco, possivelmente provenientes de reapresentação do mesmo registro ao SIA-SUS.

As variáveis utilizadas foram: 1- Neoplasia: CID definida como principal na Apac; 2- Ano da Apac: criada a partir da variável competência; 3- Habilitaçáo do estabelecimento de saúde: criada a partir do CNES do estabelecimento e das portarias de habilitação vigentes para os anos de estudo; 4- Número de campos de RxT por área irradiada; 5- Finalidade terapêutica da radioterapia: agrupada em paliativa (antiálgica, anti-hemorrágica e paliativa) e não paliativa (radical, adjuvante e prévia); 6- CNS: dado codificado; 7- Idade: variável contínua; 8Estadiamento: estádios 0, I, II, III e IV; 9- Procedimento principal: roentgenterapia, cobaltoterapia, radioterapia com acelerador linear só de fótons e radioterapia com acelerador linear de fótons e elétrons; 10- Continuidade do tratamento: (sim/não).

O tipo de tratamento de RxT realizado foi classificado a partir do procedimento principal. O total de campos por tratamento foi obtido pela variável 'número de campos de RxT por área irradiada' e os registros com valor igual a zero foram tratados como missing. $\mathrm{O}$ número máximo de campos da área tratada por acelerador linear e cobaltoterapia considerou as normas preconizadas no Manual de Bases Técnicas da Oncologia ${ }^{6}$. Para essa avaliação, foram utilizadas as Apac-RxT iniciais cujo procedimento principal informado era o acelerador linear (por fótons e por fótons e elétrons) e cobaltoterapia, pois estes possibilitam avaliar o número de campos utilizados para o tratamento de RxT. A escolha por esses procedimentos para descrever o número de campos tratados segue a descrição de produção de campos por equipamento, conforme estabelecido na Portaria 140/2014 ${ }^{8}$.

Para avaliar a finalidade da radioterapia, os códigos informados que não correspondiam às categorias previstas no dicionário do banco de dados ${ }^{11}$ foram tratados como missing.

Para obter apenas os CNS associados à produção de Apac-RxT iniciais do período 2012 a 2016, o ano de 2011 foi incluído na base de dados para identificar possíveis casos que iniciaram RxT antes do período de interesse. Esse processo foi realizado por meio da frequência dos CNS únicos e do ano das Apac-RxT iniciais. Dessa forma, foi possível excluir a produção dos CNS que constavam no banco de 2012-2016, mas que haviam iniciado seu tratamento em anos anteriores.

As bases de dados foram obtidas no sítio do Departamento de Informática do SUS (DATASUS) ${ }^{11}$, na seçâo serviços, e o download foi realizado no período de abril a maio de 2017. Foi utilizado o programa $\mathrm{R}^{12}$ versão 3.4.3 para análise dos dados.

Por terem sido utilizados dados secundários, de uso público, sem identificação dos indivíduos, tal estudo isenta-se de ser apreciado pelo Comitê de Ética em Pesquisa (CEP) do Conselho Nacional de Saúde (Conep) ${ }^{13}$.

\section{RESULTADOS}

Para o período de 2012 a 2016, foram selecionadas 620.057 autorizaçóes de Apac-RxT iniciais, correspondendo a 509.708 CNS, já excluídos os CNS e suas Apac-RxT iniciais correspondentes, cujo primeiro registro era do ano 2011. O número de CNS por ano de competência do tratamento ficou em torno de 100 mil por ano, com um incremento progressivo até 2014 (104.173 CNS); reduzindo nos anos posteriores, registrando, em 2016, 99.720 CNS novos (Figura 1).

\begin{tabular}{|c|c|c|}
\hline Ano & CNS & $\begin{array}{c}\text { Apac- } \\
\text { RxT } \\
\text { iniciais }\end{array}$ \\
\hline 2012 & 100.215 & 115.755 \\
2013 & 102.092 & 124.504 \\
2014 & 104.173 & 127.751 \\
2015 & 103.508 & 128.068 \\
2016 & 99.720 & 123.979 \\
\hline Total & $\mathbf{5 0 9 . 7 0 8}$ & $\mathbf{6 2 0 . 0 5 7}$ \\
$\quad$ & $\begin{array}{c}\text { Excluídos os CNS com ano de } \\
\text { autorização anterior a 2012 e suas } \\
\text { respectivas Apac-RxT iniciais } \\
\text { subsequentes }\end{array}$ \\
\hline
\end{tabular}

Figura 1. Número do Cartão Nacional de Saúde e autorizações iniciais por ano

Legendas: CNS: Cartão Nacional de Saúde; Apac-RxT iniciais: Autorizaçōes de Procedimentos de Alta Complexidade em Oncologia para radioterapia iniciais. Fonte: Sistema de Informação Ambulatorial/Autorização de Procedimentos de Alta Complexidade.

Do total de autorizaçóes, 412.464 CNS apareceram uma única vez; ou seja, uma Apac-RxT inicial para um CNS, e 97.244 CNS apareceram mais de 1 vez, correspondendo a duas ou mais Apac-RxT iniciais para um mesmo CNS. O total de Apac-RxT iniciais dos CNS que apareceram mais de uma vez foi de 207.593, o que resultou em uma razão 2,1 Apac-RxT iniciais/CNS (variando entre 2,0 para neoplasia maligna de estômago e 2,3 para outra neoplasia maligna de pele).

Verificou-se que, entre os 509.708 CNS do período 2012 a 2016, 11,3\% tinham registro de continuidade do tratamento. Destes, $37,1 \%$ tinham informação de tratamento anterior; $12,9 \%$ tinham registro de não ter 
realizado tratamento anterior; e, em 50,0\% dos CNS, o campo não foi preenchido.

No período analisado, as topografias mais frequentes foram as neoplasias malignas de mama (C50); próstata (C61); colo do útero (C53); pulmão (C34); reto (C20); outras neoplasias de pele (C44); esôfago (C15); corpo do útero (C54); laringe (C32); encéfalo (C71); estômago (C16); e orofaringe (C10), correspondendo a $81,4 \%$ das autorizaçōes.

Entre os 509.708 CNS, 24,3\% dos pacientes trataram neoplasia maligna de mama e $18,5 \%$ de próstata. As neoplasias com estadiamento mais avançado (estádio IV) foram orofaringe e pulmão, com 49,9\% e 48,9\%, respectivamente (Tabela 1 ).

A maioria das autorizaçôes $(85,2 \%)$ foi para finalidade não paliativa. Entre aquelas com finalidade paliativa, as neoplasias com maior proporção foram pulmáo $(42,2 \%)$ e estômago (21\%), excluídas as outras neoplasias. Apesar da alta proporção de estágio IV para as neoplasias de orofaringe, apenas $11,3 \%$ tinham finalidade paliativa. A diferença entre a proporção de Apac-RxT iniciais por finalidade paliativa e não paliativa foi significativa para todas as neoplasias, exceto para pulmão (Tabela 1).

Não foi registrada a finalidade terapêutica para 2.320 Apac-RxT iniciais $(0,5 \%)$. A proporção de não registro da finalidade terapêutica por neoplasia variou de 1,3\% para pulmáo a $0,2 \%$ para as neoplasias do colo do útero e corpo do colo do útero.
As medianas de idade foram maiores para os casos de outras neoplasias malignas de pele e de próstata (ambas com mediana $=70$ anos) e menores para colo do útero e encéfalo, 52 e 48 anos, respectivamente. A neoplasia de encéfalo apresentou maior variabilidade na distribuição da idade e foi a única que náo apresentou nenhum outlier (Figura 2).

O número médio de campos da área tratada por acelerador linear e cobaltoterapia variou conforme a neoplasia tratada e a finalidade terapêutica. $\mathrm{O}$ número médio de campos para os tratamentos com finalidade paliativa comparada à não paliativa foi maior para as neoplasias laringe, reto, orofaringe. Enquanto, para o colo do útero, foram praticamente iguais (Tabela 2). Para "outras neoplasias malignas da pele", não foi possível afirmar que o número de campos na condição paliativa foi maior que a não paliativa, já que o Manual de Bases Técnicas da Oncologia ${ }^{6}$ estabelece o limite de campos para "pele tumores epiteliais" e "pele com cadeias linfáticas".

O número máximo de campos da área tratada por acelerador linear e cobaltoterapia com finalidade não paliativa foi superior ao estabelecido para as neoplasias de mama, colo do útero, corpo do útero, pulmão, laringe, reto, estômago e esôfago (Tabela 2).

Observou-se que a maioria dos casos receberam tratamento radioterápico em estabelecimentos habilitados, como Cacon com serviços de Oncologia Pediátrica, seguido das Unacon com radioterapia. Os

Tabela 1. Neoplasias malignas mais frequentes no tratamento radioterápico no SUS por CNS segundo estadiamento e finalidade terapêutica. Brasil, 2012 a 2016

\begin{tabular}{|c|c|c|c|c|c|c|c|c|c|c|c|}
\hline \multirow{2}{*}{ Neoplasia } & \multirow{2}{*}{ CID } & \multicolumn{2}{|c|}{ Total } & \multicolumn{5}{|c|}{ Estadiamento } & \multicolumn{2}{|c|}{$\begin{array}{l}\text { Finalidade } \\
\text { terapêuticab }\end{array}$} & \multirow{2}{*}{ p-valor } \\
\hline & & $\mathbf{N}$ & $\%$ & 0 & I & II & III & IV & $\begin{array}{c}\% \\
\text { Paliativo }\end{array}$ & $\begin{array}{c}\text { \% não } \\
\text { paliativo }\end{array}$ & \\
\hline Orofaringe & $\mathrm{C} 10$ & 10.118 & 2,0 & 5,0 & 6,2 & 13,3 & 25,6 & 49,9 & 11,3 & 88,7 & $<0,001$ \\
\hline Esôfago & $\mathrm{C} 15$ & 19.838 & 3,9 & 7,0 & 6,3 & 29,4 & 43,8 & 13,5 & 16,0 & 84,0 & $<0,001$ \\
\hline Estômago & $\mathrm{C} 16$ & 10.517 & 2,1 & 6,2 & 5,0 & 19,8 & 44,4 & 24,6 & 21,0 & 79,0 & $<0,001$ \\
\hline Reto & $\mathrm{C} 20$ & 23.217 & 4,6 & 8,2 & 6,1 & 32,4 & 38,5 & 14,8 & 14,7 & 85,3 & $<0,001$ \\
\hline Laringe & $\mathrm{C} 32$ & 17.242 & 3,4 & 5,0 & 17,3 & 18,4 & 26,0 & 33,3 & 7,2 & 92,8 & $<0,001$ \\
\hline Pulmão & C34 & 22.762 & 4,5 & 7,2 & 3,7 & 9,5 & 30,7 & 48,9 & 42,2 & 57,8 & 0,119 \\
\hline Pele & $\mathrm{C} 44$ & 17.592 & 3,4 & 7,0 & 38,4 & 28,7 & 16,1 & 9,8 & 6,5 & 93,5 & $<0,001$ \\
\hline Mama & C50 & 123.750 & 24,3 & 8,6 & 19,4 & 30,5 & 29,5 & 12,0 & 10,6 & 89,4 & $<0,001$ \\
\hline Colo do útero & $\mathrm{C} 53$ & 49.094 & 9,6 & 6,3 & 15,1 & 31,3 & 35,5 & 11,8 & 7,9 & 92,1 & $<0,001$ \\
\hline $\begin{array}{l}\text { Corpo do } \\
\text { útero }^{a}\end{array}$ & C54 & 12.130 & 2,4 & 8,9 & 34,9 & 24,9 & 20,3 & 11,0 & 8,4 & 91,6 & $<0,001$ \\
\hline Próstata & C61 & 94.495 & 18,5 & 8,3 & 15,6 & 47,9 & 16,4 & 11,8 & 12,6 & 87,4 & $<0,001$ \\
\hline Encéfalo & C71 & 10.811 & 2,1 & NA & NA & NA & NA & NA & 11,7 & 88,3 & $<0,001$ \\
\hline \multicolumn{2}{|c|}{ Outras neoplasias ${ }^{a}$} & 98.142 & 19,2 & 7,9 & 8,9 & 21,1 & 24,7 & 37,4 & 22,9 & 77,1 & $<0,001$ \\
\hline Total & & 509.708 & 100,0 & & & & & & 14,8 & 85,2 & $<0,001$ \\
\hline
\end{tabular}

Fonte: Sistema de Informação Ambulatorial/Autorização de Procedimentos de Alta Complexidade.

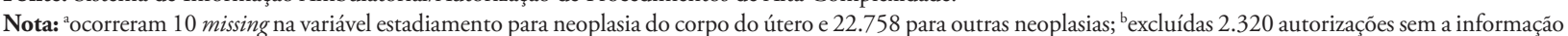
da finalidade da RxT; ' p-valor qui-quadrado para homogeneidade da distribuiçáo (finalidade terapêutica); NA: não se aplica. 


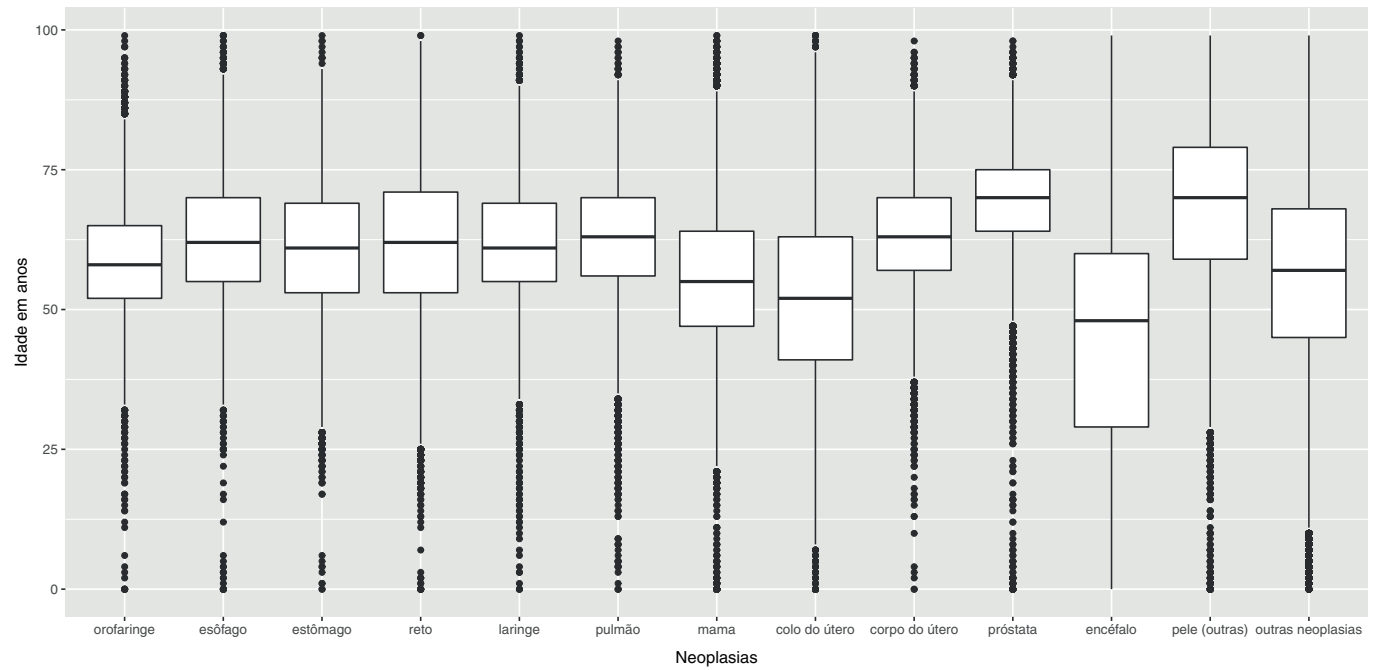

Figura 2. Mediana de idade dos casos que realizaram tratamento radioterápico no SUS pelas neoplasias mais frequentes. Brasil, 2012 a 2016 Fonte: Sistema de Informaçăo Ambulatorial/Autorizaçāo de Procedimentos de Alta Complexidade.

Tabela 2. Distribuição dos CNS submetidos a tratamento radioterápico por acelerador linear e cobaltoterapia no SUS, segundo número máximo de campos, campos médio, mediano, máximo e $3^{\circ}$ quartil por finalidade terapêutica. Brasil, 2012 a 2016

\begin{tabular}{|c|c|c|c|c|c|c|c|c|c|c|c|c|c|c|}
\hline \multirow[b]{2}{*}{ Neoplasia } & \multirow{2}{*}{$\begin{array}{c}\text { Número } \\
\text { máximo de } \\
\text { campos }^{1}\end{array}$} & \multicolumn{6}{|c|}{ Finalidade paliativa } & \multicolumn{6}{|c|}{ Finalidade não paliativa } & \multirow[b]{2}{*}{$p$-valor } \\
\hline & & $\begin{array}{l}\text { Total de } \\
\text { CNS }\end{array}$ & Mediana & Média & $3^{\circ} \mathrm{Qt}$. & Max. & $\%$ NA & $\begin{array}{l}\text { Total de } \\
\text { CNS }\end{array}$ & Mediana & Média & $3^{\circ} \mathrm{Qt}$. & Max. & $\%$ NA & \\
\hline Mama & 120 & 13.017 & 32 & 43 & 75 & 120 & 9,0 & 110.346 & 56 & 62 & 120 & 144 & 8,7 & 0,94 \\
\hline Próstata & 140 & 11.861 & 40 & 61 & 144 & 144 & 7,6 & 82.230 & 132 & 79 & 144 & 144 & 7,4 & 0,96 \\
\hline Colo do útero & 120 & 3.335 & 60 & 65 & 120 & 120 & 8,2 & 38.814 & 100 & 65 & 120 & 140 & 5,8 & 0,52 \\
\hline Corpo do útero & 110 & 878 & 40 & 56 & 108 & 120 & 11,0 & 7.501 & 92 & 60 & 110 & 120 & 7,9 & 0,48 \\
\hline Outras neoplasias de pele & NA & 1.118 & 25 & 24 & 30 & 120 & 12,7 & 12.312 & 25 & 22 & 30 & 144 & 4,8 & 0,06 \\
\hline Pulmão & 90 & 9.491 & 20 & 32 & 60 & 120 & 7,5 & 12.979 & 40 & 46 & 90 & 144 & 4,4 & 0,37 \\
\hline Laringe & 105 & 1.231 & 80 & 62 & 105 & 120 & 10,4 & 15.954 & 50 & 52 & 105 & 144 & 4,7 & 0,14 \\
\hline Reto & 100 & 3.406 & 75 & 59 & 100 & 120 & 6,6 & 19.749 & 56 & 54 & 100 & 140 & 6,0 & 0,87 \\
\hline Estômago & 60 & 2.202 & 20 & 29 & 60 & 80 & 5,4 & 8.277 & 40 & 33 & 60 & 120 & 4,8 & 0,85 \\
\hline Esôfago & 105 & 3.156 & 40 & 52 & 105 & 120 & 12,0 & 16.552 & 56 & 54 & 105 & 144 & 5,2 & 0,10 \\
\hline Orofaringe & 105 & 1.141 & 66 & 57 & 105 & 105 & 10,0 & 8.934 & 55 & 55 & 105 & 105 & 4,9 & 0,19 \\
\hline Encéfalo & 70 & 1.255 & 39 & 40 & 70 & 120 & 8,7 & 9.504 & 70 & 56 & 90 & 120 & 4,7 & 0,27 \\
\hline Outras neoplasias & & 22.091 & 20 & 34 & 40 & 144 & 10,0 & 71.459 & 40 & 46 & 90 & 144 & 6,0 & 0,31 \\
\hline
\end{tabular}

Fonte: Sistema de Informação Ambulatorial/Autorização de Procedimentos de Alta Complexidade.

Nota: ${ }^{1}$ Limite estabelecido no Manual de Bases Técnicas da oncologia - SIA/SUS; excluídos 2.278 procedimentos por acelerador linear e cobalto sem finalidade terapêutica; NA: Não se aplica.

estabelecimentos habilitados como Cacon apresentaram um número médio de CNS maior (4,7 a 6 mil CNS por estabelecimento habilitado), quando comparado à Unacon (abaixo de 3,7 mil CNS por estabelecimento) Tabela 3.

Destaca-se que, no Cacon, $25,9 \%$ dos tratamentos foram com finalidade paliativa, enquanto, nas Unacon Exclusiva de Oncologia Pediátrica com Serviço de Radioterapia, esta não chegou a 3\%. A finalidade 'não paliativa' variou entre 74,1 e $97,4 \%$ entre os tipos de habilitaçôes (Tabela 3).

\section{DISCUSSÃO}

Conhecer a demanda por tratamento radioterápico de pacientes diagnosticados com câncer tem sido apontado como um marcador importante da organização do acesso aos serviços de saúde com vistas à integralidade do cuidado ${ }^{4}$. Estima-se que, em países de baixa e média rendas, $50 \%$ dos casos que necessitam de tratamento por radioterapia não têm esse acesso ${ }^{14}$.

Estudo realizado para o Brasil, baseado em fontes oficiais, apontou que havia um déficit de 255 serviços de 
Tabela 3. Distribuição dos CNS submetidos a tratamento radioterápico no SUS por tipo de estabelecimento habilitado e finalidade terapêutica. Brasil, 2012 a 2016

\begin{tabular}{|c|c|c|c|c|c|c|c|}
\hline \multirow{2}{*}{$\begin{array}{l}\text { Tipo de habilitação } \\
\text { em Oncologia }\end{array}$} & \multirow{2}{*}{$\begin{array}{l}\text { Total de } \\
\text { CNS }^{b}\end{array}$} & \multirow{2}{*}{$\begin{array}{c}\text { Estabelecimentos } \\
\text { habilitados com } \\
\text { informação }\end{array}$} & \multirow{2}{*}{$\begin{array}{l}\text { Número de CNS } \\
\text { dividido por } \\
\text { estabelecimento } \\
\text { habilitado }\end{array}$} & \multicolumn{2}{|c|}{ Não paliativa } & \multicolumn{2}{|c|}{ Paliativa } \\
\hline & & & & $\mathbf{n}^{\mathrm{c}}$ & $\%$ & $\mathbf{n}^{\mathrm{c}}$ & $\%$ \\
\hline Cacon & 84.590 & 18 & 4.699 & 62.208 & $74,1 \%$ & 21.718 & $25,9 \%$ \\
\hline $\begin{array}{l}\text { Cacon com Serviço de } \\
\text { Oncologia Pediátrica }\end{array}$ & 162.587 & 27 & 6.022 & 144.960 & $89,7 \%$ & 16.707 & $10,3 \%$ \\
\hline $\begin{array}{l}\text { Unacon com Serviços de } \\
\text { Radioterapia }\end{array}$ & 123.877 & 56 & 2.212 & 106.981 & $86,6 \%$ & 16.570 & $13,4 \%$ \\
\hline $\begin{array}{l}\text { Unacon com Serviços } \\
\text { de Radioterapia e de } \\
\text { Hematologia }\end{array}$ & 71.372 & 32 & 2.230 & 59.099 & $82,9 \%$ & 12.149 & $17,1 \%$ \\
\hline $\begin{array}{l}\text { Unacon com Serviços } \\
\text { de Radioterapia e de } \\
\text { Oncologia Pediátrica }\end{array}$ & 11.156 & 3 & 3.719 & 9.389 & $84,6 \%$ & 1.709 & $15,4 \%$ \\
\hline $\begin{array}{l}\text { Unacon com Serviços } \\
\text { de Radioterapia, de } \\
\text { Hematologia e de } \\
\text { Oncologia Pediátrica }\end{array}$ & 29.563 & 11 & 2.688 & 26.392 & $89,9 \%$ & 2.949 & $10,1 \%$ \\
\hline $\begin{array}{l}\text { Unacon Exclusiva de } \\
\text { Oncologia Pediátrica } \\
\text { com Serviço de } \\
\text { Radioterapia } \\
\end{array}$ & 3.195 & 2 & 1.598 & 3.113 & $97,4 \%$ & 82 & $2,6 \%$ \\
\hline Isolada de Radioterapia ${ }^{a}$ & 23.039 & 11 & 2.094 & 19.820 & $86,1 \%$ & 3.213 & $13,9 \%$ \\
\hline Total & 509.379 & 160 & 3.184 & 431.962 & $85,2 \%$ & 75.097 & $14,8 \%$ \\
\hline
\end{tabular}

Fonte: Sistema de Informação Ambulatorial/Autorização de Procedimentos de Alta Complexidade.

Nota: ${ }^{a}$ serviço não habilitado, mas autorizado a realizar radioterapia; bexcluídos 329 CNS $(0,07 \%)$ informados por dois estabelecimentos habilitados no final de 2016 e por dois estabelecimentos que eram habilitados até 2012; 'excluídos os CNS sem informação da finalidade terapêutica.

radioterapia no país em 2015 e uma projeção de déficit de 198 serviços para $2030^{15}$, utilizando como base da estimativa uma base populacional. O presente estudo contribuiu fundamentalmente para o planejamento do acesso ao tratamento radioterápico ao identificar nas Apac em oncologia de radioterapia o número de pacientes em tratamento por tipo de neoplasia, estadiamento e o total de Apac-RxT iniciais utilizadas no tratamento. Datta et al. ${ }^{5}$ chamam a atenção para a importância de cada país conhecer sua taxa de utilização de radioterapia, a partir dos tipos de câncer e estadiamento, antes de estimar a necessidade atual e futura de serviços.

Entre as principais neoplasias malignas tratadas com RxT, encontram-se as mais incidentes no país ${ }^{16}$ : câncer de mama, próstata, colo do útero, pulmão e estômago. Esses também foram os tipos de neoplasias mais frequentes no Registro Hospitalar de Câncer no período entre 2007 e $2011^{17}$; e, excetuando estômago, estão entre as principais neoplasias informadas em cuidados paliativos na atenção domiciliar ${ }^{18}$.

O estadiamento avançado foi superior a $50 \%$ dos casos para pulmão, orofaringe, laringe, esôfago, estômago e reto. Em levantamento realizado em $2011^{17}$, sobre o tratamento oncológico, não específico de RxT, dos casos que chegaram aos estabelecimentos habilitados no SUS, brônquios e pulmóes também foram referidos como os que deram entrada com estádios mais avançados.

Contudo, ainda que a maioria dos casos submetidos ao tratamento radioterápico estivesse em estadiamento avançado, os com finalidade não paliativa representaram mais de $85 \%$ dos casos, muito superior ao observado entre os serviços públicos de radioterapia da Austrália, onde $57 \%$ foram não paliativos ${ }^{19}$. Essa diferença pode estar relacionada a diferenças na classificação da finalidade terapêutica, mas carece de outros estudos que permitam descartar erros na informação da finalidade terapêutica na Apac.

A idade mediana dos casos de mama tratados por radioterapia foi de 55 anos, próximo ao encontrado por Grantzau et al. ${ }^{20}$ cuja mediana foi de 54 anos. Bantema-Joppe et al. ${ }^{21}$ encontraram idade mediana um pouco superior, de 59 anos, em mulheres que tiveram câncer de mama estádios 0 a III e foram tratadas com cirurgia conservadora e radioterapia. Já a idade mediana dos casos câncer do colo do útero ficou próxima ao estudo de Ferrigno e Nadalin ${ }^{22}$, o qual encontrou idade mediana de 53 anos para mulheres tratadas exclusivamente com telecobalto e braquiterapia de baixa dose. Outro estudo ${ }^{23}$, 
que avaliou dois períodos distintos, encontrou idade mediana de 53 anos no período entre 1992 e 1999 e de 55 anos entre 1999 e 2005.

Para câncer de próstata, Wu et al. ${ }^{24}$ e Hashine et al. ${ }^{25}$ relatam uma idade mediana de 78 e 79 anos, respectivamente maiores que as encontrada no presente estudo (70 anos).

Um estudo com sobreviventes de tumores cerebrais ${ }^{26}$ encontrou uma idade mediana ao diagnóstico de 42 anos, menor do que a referida no presente estudo, sendo que $80 \%$ destes receberam tratamento radioterápico. Já Yersal ${ }^{27}$ refere uma idade mediana maior do que a que encontrada neste estudo, 57 anos; todavia a população do estudo era referente a pacientes com glioblastoma multiforme, dos quais apenas $5,1 \%$ receberam tratamento adjuvante por radioterapia.

As diferenças entre as medianas de idade precisam ser melhor investigadas, considerando a fonte de informação utilizada e a qualidade dos registros, a população dos diferentes estudos, o ano do estudo, a rede de serviços de saúde e a infraestrutura local disponível.

O número médio de campos de RxT variou de acordo com a neoplasia e a finalidade terapêutica. Chamou a atenção o número de campos de radioterapia e de finalidade terapêutica sem informação (missings), os quais são importantes para o monitoramento do tratamento e, vale ressaltar, de preenchimento obrigatório para autorizar as $A \mathrm{pac}^{6}$. Portanto, uma consideração importante a esse sistema de informação está na ausência efetiva de críticas a campos obrigatórios relacionados à qualidade do tratamento ofertado.

O número máximo de campos informado nas Apac-RxT iniciais por neoplasia não segue o limite máximo definido no Manual de Bases Técnicas da Oncologia, sinalizando para a não conformidade com os parâmetros de tratamento definidos. Cabe destacar que o número de campos pode ser superior ao estabelecido quando se tratar de RxT de metástase, porém esse campo náo está disponível na base da Apac, impossibilitando sua avaliação. Considerando esses achados, seria importante realizar avaliaçóes locais para esclarecer porque as Apac que ultrapassam o número de campos estão sendo autorizadas.

A avaliação da produção de radioterapia por tipo de unidade habilitada ao SUS é importante para monitorar a oferta desses serviços frente à necessidade. Considerando-se que 60\% dos casos novos de câncer irão necessitar de $\mathrm{RxT}^{2,3}$ e a estimativa ${ }^{16}$ de 417.010 casos novos, exceto pele não melanoma, para 2018, 250.206 mil novos pacientes/ano necessitariam de RxT. O presente estudo encontrou um incremento no tratamento de RxT de 100 mil pacientes/ano, o que sugere possível não absorção de uma demanda de mais de $50 \%$ dos que necessitam.
Essa demanda reprimida vai ao encontro da proporção estimada por Zubizarreta et al. ${ }^{14}$. Quando se avalia a produção dos estabelecimentos habilitados em oncologia é importante considerar a capacidade instalada de cada um deles, que é dada pelo número de aceleradores lineares e unidades de cobaltoterapia. Isso justifica a maior produção de RxT encontrada nos estabelecimentos habilitados como Cacon que possuem maior capacidade instalada quando comparados à Unacon.

O estudo levanta questốes relevantes para a gestáo, o planejamento, a avaliação e o monitoramento do cuidado ao paciente oncológico, que precisam ser abordadas tendo em vista a primazia pela qualidade da assistência prestada. Para tanto, é necessário avaliar o que representa um número total de campos superior ao preconizado e equacionar a proporção de estadiamentos avançados e de tratamentos com finalidade paliativa, bem como avaliar a capacidade de absorção de novos casos frente ao incremento observado e a uma possível náo absorção de $50 \%$ da demanda de necessidade por radioterapia.

A escassez de estudos semelhantes a este trabalho reitera a sua relevância e, ao mesmo tempo, limita a discussão quanto à comparabilidade dos resultados apresentados. $\mathrm{E}$ ainda sinaliza a necessidade de pesquisas que forneçam informaçôes que subsidiem a gestão na organização da oferta do tratamento oncológico.

Embora não tenha sido objetivo desse estudo avaliar a qualidade dos dados disponíveis na Apac, não há como furtar-se a observar o potencial que tais informaçóes têm para o planejamento e gestão do tratamento oncológico, apesar da fragilidade do sistema quanto à ausência de crítica em algumas variáveis. Muito do que está estabelecido no Manual de Bases Técnicas da Oncologia ${ }^{6}$ não é observado nos bancos de dados, o que fragiliza as análises e dificulta o acompanhamento dos casos de câncer submetido a essa modalidade terapêutica. Ainda assim, alguns dos achados no presente estudo encontram suporte na literatura no que se refere aos casos mais frequentes, à faixa etária e ao estadiamento.

\section{CONCLUSÃO}

A radioterapia está presente em grande parte dos planos terapêuticos dos pacientes oncológicos e, de acordo com presente estudo, houve um incremento de $20 \%$ no número de casos que demandaram tratamento radioterápico. Assim, constata-se que urge um planejamento capaz de garantir tanto o acesso a essa modalidade terapêutica quanto a qualidade do tratamento prestado.

Para tanto, é importante mais estudos que se dediquem a descrever tanto o cenário epidemiológico quanto o tratamento ofertado aos pacientes com câncer para 
subsidiar a gestáo na organização da rede de atenção à saúde observando os princípios do SUS.

\section{CONTRIBUIÇÕES}

Jeane Glaucia Tomazelli contribuiu com a concepção e delineamento do estudo, análise e interpretaçáo dos dados, redação e revisão crítica relevante do conteúdo intelectual do manuscrito. Adriana Tavares de Moraes Atty contribuiu com a concepção e delineamento do estudo, extração, análise e interpretação dos dados, redação e revisão crítica relevante do conteúdo intelectual do manuscrito. Antônio Carlos Antunes Bertholasce colaborou na análise e interpretação dos dados e revisão crítica relevante do conteúdo intelectual do manuscrito. Maria Beatriz Kneipp Dias colaborou na análise e interpretação dos dados, redação e revisão crítica relevante do conteúdo intelectual do manuscrito. Todos os autores aprovaram a versão final do manuscrito e declaram serem responsáveis por todos os aspectos do trabalho, garantindo sua precisão e integridade.

\section{DECLARAÇÃO DE CONFLITO DE INTERESSES}

Nada a declarar.

\section{FONTES DE FINANCIAMENTO}

Não há.

\section{REFERÊNCIAS}

1. Steward BW, Wild CP, editors. World Cancer Report 2014. Lyon: IARC; 2014. 630 p.

2. International Atomic Energy Agency (AT). Planning national radiotherapy services: a practical tool. Vol. 14. Vienna: International Atomic Energy Agency; 2011. 83 p. (IAEA Human Health Series; no. 14).

3. Zubizarreta EH, Fidarova E, Healy B, Rosenblatt E. Need for radiotherapy in low and middle income countries - the silent crisis continues. Clin Oncol. 2015 Feb;27(2):107-14.

4. Rosenblatt E, Barton M, Mackillop W, Fidarova E, Cordero L, Yarney J, et al. Optimal radiotherapy utilisation rate in developing countries: an IAEA study. Radiother Oncol. 2015 Jul;116(1):35-7.

5. Datta NR, Samiei M, Bodis S. Radiation therapy infrastructure and human resources in low- and middleincome countries: present status and projections for 2020. Int J Radiat Oncol Biol Phys. 2014 Jul;89(3):448-57.

6. Ministério da Saúde (BR), Secretaria de Atenção à Saúde. Manual de bases técnicas da oncologia - SIA/SUS: sistema de informaçôes ambulatoriais. 19 ed. Brasília, DF: Ministério da Saúde; 2015. 128 p.
7. Instituto Nacional de Câncer. Açóes de enfermagem para o controle do câncer: uma proposta de integração ensino-serviço. 3. ed., atual., ampl. Rio de Janeiro: INCA; 2008.

8. Ministério da Saúde (BR). Portaria no 140 , de 27 de fevereiro de 2014. Redefine os critérios e parâmetros para organizaçáo, planejamento, monitoramento, controle e avaliação dos estabelecimentos de saúde habilitados na atençấo especializada em oncologia e define as condiçôes estruturais, de funcionamento e de recursos humanos para a habilitação destes estabelecimentos no âmbito do Sistema Único de Saúde (SUS). Diário Oficial da União, Brasília, DF; 28 Fev 2014.

9. Instituto Nacional de Câncer. Programa de qualidade em radioterapia: manual para técnicos em radioterapia. Rio de Janeiro: INCA; 2000.

10. Ministério da Saúde (BR), Secretaria de Atençâo à Saúde. Manual técnico operacional SIA/SUS sistema de informaçôes ambulatoriais: aplicativos auxiliares e de captação da produção ambulatorial: APAC Magnético - BPA Magnético: VERSIA - DE-PARA - FPO Magnético: orientaçôes técnicas. Brasília, DF: Ministério da Saúde; 2010. 69 p.

11. Ministério da Saúde (BR), DATASUS, CoordenaçãoGeral de Disseminação de Informaçôes em Saúde. Disseminação de informaçôes em saúde: sistema de informaçóes ambulatoriais do SUS - SIASUS: Informe Técnico [Internet]. Rio de Janeiro: Coordenação-Geral de Disseminação de Informações em Saúde. [data desconhecida] - [acesso 2018 May 6]. Disponível em: ftp:// ftp.datasus.gov.br/dissemin/publicos/SIASUS/200801_/ Doc/Informe_Tecnico_SIASUS_2018-05.pdf

12. R Foundation for Statistical Computing. The R Project for Statistical Computing. [computer program]. R version 3.4.3. [Internet]. [place unknown]: The $\mathrm{R}$ foundation; 2017. [cited 2017 Jul 15]. Available from: https://www.R-project.org.

13. Conselho Nacional de Saúde (BR). Resolução no 466, de 12 de dezembro de 2012 [Internet]. [acesso 2017 Ago 30]. Disponível em: http://conselho.saude.gov.br/ resolucoes/2012/Reso466.pdf

14. Zubizarreta E, Van Dyk J, Lievens Y. Analysis of global radiotherapy needs and costs by geographic region and income level. Clin Oncol. 2017 Feb;29(2):84-92.

15. Araújo LP, Sá NM, Atty ATM. Necessidades atuais de radioterapia no SUS e estimativas para o ano de 2030. Rev Bras Cancerol. 2016;62(1):35-42.

16. Instituto Nacional de Câncer José de Alencar Gomes da Silva. Estimativa 2018: incidência de câncer no Brasil. Rio de Janeiro: INCA; 2017.

17. Instituto Nacional do Câncer José de Alencar Gomes da Silva. Perfil da assistência oncológica no Brasil, de 2007 a 2011. Informativo Vigilância do Câncer. 2015 Jan/Jul; (6):1-12. 
18. Atty ATM, Tomazelli JG. Cuidados paliativos na atenção domiciliar para pacientes oncológicos no Brasil. Saude Debate. 2018 Jan-Mar;42(116):225-236.

19. Australian Institute of Health and Welfare. Radiotherapy in Australia: report on a pilot data collection 2013-14 [Internet]. [cited 2017 Ago 30]. Canberra: AIHW; 2015. Available from: https:/www.aihw.gov.au/reports/ hospitals/radiotherapy-in-australia-2013-14/contents/ table-of-contents.

20. Grantzau T, Thomsen MS, Vaeth M, Overgaard J. Risk of second primary lung cancer in women after radiotherapy for breast cancer. Radiother Oncol. 2014 Jun;111(3):366-73.

21. Bantema-Joppe EJ, de Bock GH, Woltman-van Iersel M, Busz DM, Ranchor AV, Langendijk JA, et al. The impact of age on changes in quality of life among breast cancer survivors treated with breast-conserving surgery and radiotherapy. Br J Cancer. 2015 Feb 17;112(4):636-43.

22. Ferrigno R, Nadalin, W. Radioterapia exclusiva no tratamento do câncer do colo do útero com telecobalto e braquiterapia de baixa dose: análise de resultados e variáveis. [Resumo de Tese]. Radiol Bras 2001;34(6):368.

23. Samant R, Kobeleva S, Choan E, Balaraj K, Le T, FungKee-Fung M. Evaluation contemporary radiotherapy approaches treatment of cervical cancer. Int J Gynecol Cancer. 2010 Aug;20(6):1087-91.

24. Wu Y-H, Yang W-C, Hu Y-W, Hsieh C-M, Yang K-L, Lai I-C, et al. Definitive radiotherapy for older patients with prostate cancer: experience of a medical center in Taiwan. Sci Rep [Internet]. 2017 Oct [cited 2017 Nov 14];7(1). Available from: http://www.nature.com/articles/s41598017-13119-3

25. Hashine K, Azuma K, Koizumi T, Sumiyoshi Y. Healthrelated quality of life and treatment outcomes for men with prostate cancer treated by combined external-beam radiotherapy and hormone therapy. Int J Clin Oncol. 2005 Feb;10(1):45-50.

26. Sloane K, Metz JM, Vachani C, Hampshire MK, HillKayser CE. Patient reported outcomens after radiation therapy and other treatments for primary brain tumors. Int J Radiat Oncol Biol Phys. 2015 Nov [cited 2017 Ago 14];93(Supp 3):E102. Available from: www.redjournal. org/article/S0360-3016(15)01539-4/fulltext

27. Yersal Ö. Clinical outcome of patients with glioblastoma multiforme: single center experience. Journal of Oncological Sciences. 2017 Dec; 3(3):123-126. 